\title{
Cannabis cures the spine
}

\author{
Arnar Geirsson, MD
}

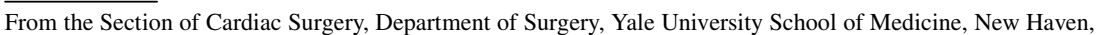
Conn.

Disclosures: Author has nothing to disclose with regard to commercial support.

Received for publication July 29, 2018; accepted for publication July 30, 2018; available ahead of print Aug 29, 2018.

Address for reprints: Arnar Geirsson, MD, Section of Cardiac Surgery, Department of Surgery, Yale University School of Medicine, 330 Cedar St, Boardman 204, New Haven, CT 06510 (E-mail: arnar.geirsson@ yale.edu). J Thorac Cardiovasc Surg 2019;157:506-7

0022-5223/\$0.00

Published by Elsevier Inc. on behalf of The American Association for Thoracic Surgery https://doi.org/10.1016/j.jtcvs.2018.07.087

Paraplegia remains among most feared complication of descending and thoracoabdominal aortic surgery. It results in significant long-term debilitation and is associated with high rates of complications as well as increased hospital cost. In addition to meticulous surgical techniques and performing the operation in an expedious manor, various adjunctive methods are used in attempts to decrease the rates of spinal ischemia during complex cases. This includes left heart bypass, moderate systemic hypothermia, improving spinal perfusion by spinal drainage and permissive hypertension, reimplantation of spinal arterial supply, localized cooling of the spinal cord, and intrathecal papaverine. $^{1-3}$ Systemic pharmacologic agents such as steroids, naloxone, and barbiturates are commonly used, although strong clinical evidence supporting their use remains limited. Identification of a novel systemic agent effective for spinal cord protection would be a significant breakthrough and of great value to clinicians taking care of patients undergoing these complex operations.

The endocannabinoid system is involved in control of neuronal function and inflammation. It is composed of cannabinoid receptor (CB) 1 and CB2 and endogenous ligands anandimide and 2-arachidonoylglycerol. They mediate synaptic plasticity and progenitor cell fate in the central nervous system and there is reasonable evidence that the endocannabinoid system is important in neuron injury. ${ }^{4}$

Huo and colleagues ${ }^{5}$ elegantly demonstrate that the endogenous cannabinoid system can be modulated to provide neuroprotection in ischemic injury of the spine. They used an established spinal cord ischemia/reperfusion model in rats and administered the synthetic cannabinoid agonist WIN55212-2 systematically. This provided neuroprotective effects that appear to be mediated through glyceraldehyde-3-phosphate dehydrogenase 7 in absentia homolog-1 (GAPDH/Siah1) inhibition. More specifically, rats treated with WIN55212-2 had significantly better neurologic function compared with the control group and

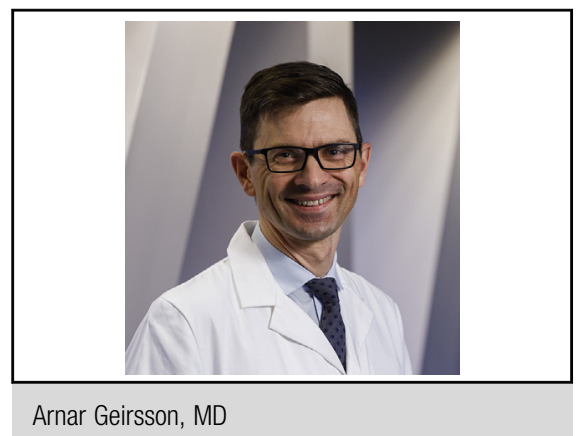

Central Message

Modulation of the endocannabinoid system attenuates ischemic spinal cord injury through CB2-mediated inhibition of the GAPDH/ Siah1 signaling cascade, positively influencing neuron survival and function.

See Article page 494

evidence of improved neuron survival after ischemic injury. GAPDH levels, nuclear translocation, and generation of the GAPDH/Siah1 complex increases following injury of the spinal cord. This is assocated with apoptosis and localized inflammatory response. Through CB2, WIN55212-2 effectively decreased the nuclear expression and complexing of GAPDH/Siah1 and reduced the production of proinflammatory cytokines interleukin $1 \beta$ and tumor necrosis factor $\alpha$ as well as nuclear factor $\kappa \mathrm{B}$, a key mediator or inflammation and decreased the level of apoptosis in the spine.

Cannabinoids are a diverse class of compounds that act on CB1 and CB2. The best known cannabinoid is tetrahydrocannabinal, the primary compound found in cannabis. There is reasonable evidence that cannabinoids can be used effectively to treat chronic pain and reduce muscle spasms in multiple sclerosis and paraplegia. There is also evidence that the compounds have moderate beneficial effects in chemotherapy-related nausea, sleep disorders, and Tourette syndrome. ${ }^{6}$ What role cannabinoids - in the form of medical marijuana, extracts, or synthetic agonistshave in treatment of various diseases is hotly debated in the medical community. ${ }^{7}$ Although the current results are promising, further research is required to determine whether CB2 agonists have any significant neuroprotective effects beyond this animal model and whether they could be used as an adjunct to decrease the risk of paraplegia following thoracic aneurysm surgery. 


\section{References}

1. Bickness CD, Riga CV, Wolfe JHN. Prevention of paraplegia during thoracoabdominal aortic aneurysm repair. Eur J Vasc Endovasc Surg. 2009;37:654-60.

2. Lima B, Novicki ER, Blackstone EH, Williams SJ, Roselli EE, Sabik JF, et al. Spinal cord protective strategies during descending and thoracoabdominal aortic aneurysm repair in the modern era: the role of intrathecal papaverine. J Thorac Cardiovasc Surg. 2012;143:945-52.

3. Etz CD, Weigang E, Hartert M, Lonn L, Mestres CA, DiBartolomeo R, et al. Contemporary spinal cord protection during thoracic and thoracoabdominal aortic surgery and endovascular aortic repair: a position paper of the vascular domain of the European Association for Cardio-Thoracic Surgery. Eur J Cardiothorac Surg. 2015;47:943-57.
4. Garcia-Arencibia M, Molina-Holgado E, Molina-Holgado F. Effect of endocannabinoid signalling on cell fate: life, death, differentiation and proliferation of brain cells. Br J Pharmacol. May 24, 2018 [Epub ahead of print].

5. Hou J, Ma R, Chai X, Liang HJ, Jiang P, Zhu XL, et al. Inhibiting a spinal cord signaling pathway protects against ischemia injury in rats. J Thorac Cardiovasc Surg. 2019;157:494-503.e1.

6. Whiting PF, Wolff RF, Deshpande S, DiNisio M, Duffy S, Hernandez AV, et al. Cannabinoids for medical use: a systematic review and meta-analysis. JAMA 2015;313:2456-73.

7. Farrell M, Buchbinder R, Hall W. Should doctors prescribe cannabinoids. BMJ. 2014;384:2737. 